PROCEEDINGS OF THE

AMERICAN MATHEMATICAL SOCIETY

Volume 130, Number 4, Pages 1243-1246

S 0002-9939(01)06191-3

Article electronically published on October 1, 2001

\title{
POWERS OF $\mathbb{N}^{*}$
}

\author{
ILIJAS FARAH
}

(Communicated by Alan Dow)

\begin{abstract}
We prove that the Čech-Stone remainder of the integers, $\mathbb{N}^{*}$, maps onto its square if and only if there is a nontrivial map between two of its different powers, finite or infinite. We also prove that every compact space that maps onto its own square maps onto its own countable infinite product.
\end{abstract}

The structure of the Čech-Stone remainder

$$
\mathbb{N}^{*}=\beta \mathbb{N} \backslash \mathbb{N}
$$

of the integers is very sensitive to the choice of set-theoretic axioms (see [4]). For instance, the Continuum Hypothesis implies that $\mathbb{N}^{*}$ maps onto $\left(\mathbb{N}^{*}\right)^{\kappa}$ for all $\kappa \leq \mathfrak{c}$ ([5]), but under different axioms $\mathbb{N}^{*}$ does not even map onto $\left(\mathbb{N}^{*}\right)^{2}$ and moreover, $\left(\mathbb{N}^{*}\right)^{n}$ does not map onto $\left(\mathbb{N}^{*}\right)^{n+1}$ for any $n \in \mathbb{N}([2])$. Obviously, if $\mathbb{N}^{*}$ maps onto $\left(\mathbb{N}^{*}\right)^{2}$, then $\left(\mathbb{N}^{*}\right)^{n}$ maps onto $\left(\mathbb{N}^{*}\right)^{n+1}$ for all $n \in \mathbb{N}$. This suggests the following question:

Question 1 (Just [2, p. 60]). Is it relatively consistent with the usual axioms of Set Theory that there are $m>n$ in $\mathbb{N}$ such that $\left(\mathbb{N}^{*}\right)^{m}$ maps onto $\left(\mathbb{N}^{*}\right)^{m+1}$, but $\left(\mathbb{N}^{*}\right)^{n}$ does not map onto $\left(\mathbb{N}^{*}\right)^{n+1}$ ?

The following theorem implies that the answer is (rather surprisingly) negative.

Theorem 2. The following are equivalent ( $X \rightarrow Y$ stands for " $X$ maps onto $Y$ "):

(1) $\mathbb{N}^{*} \rightarrow\left(\mathbb{N}^{*}\right)^{2}$.

(2) $\left(\mathbb{N}^{*}\right)^{n} \rightarrow\left(\mathbb{N}^{*}\right)^{n+1}$ for some $n \in \mathbb{N}$.

(3) $\left(\mathbb{N}^{*}\right)^{\kappa} \rightarrow\left(\mathbb{N}^{*}\right)^{\lambda}$ for some pair of cardinals $\kappa<\lambda$, finite or infinite.

(4) $\mathbb{N}^{*} \rightarrow\left(\mathbb{N}^{*}\right)^{\mathbb{N}}$.

The key behind the proof of Theorem 2 is in [1, Theorem 3] (see Theorem [5 below), where it was proved that every map from a power of $\mathbb{N}^{*}$ into $\mathbb{N}^{*}$ essentially depends on at most one coordinate.

The clauses (1) and (4) are equivalent for all compact spaces.

Theorem 3. A compact space $X$ maps onto $X^{2}$ if and only if it maps onto $X^{\mathbb{N}}$.

Received by the editors August 10, 2000 and, in revised form, October 31, 2000.

2000 Mathematics Subject Classification. Primary 54B10, 54D30, 54D35, 54C05.

Key words and phrases. Čech-Stone compactifications, product spaces, continuous images.

The author acknowledges support received from the National Science Foundation (USA) via grant DMS-0070798 and from the PSC-CUNY grant. 
We also prove another surprising fact about powers of $\mathbb{N}^{*}$.

Theorem 4. There is a cardinal $\mu$ such that for every pair of cardinals $\kappa, \lambda$ the following are equivalent:

(1) $\left(\mathbb{N}^{*}\right)^{\kappa} \rightarrow\left(\mathbb{N}^{*}\right)^{\lambda}$.

(2) $\kappa \geq \lambda$ or $\kappa<\lambda<\mu$.

We start by recounting terminology and some results of [1]. If $s \subseteq \kappa$, then $\pi_{s}^{\kappa}: X^{\kappa} \rightarrow X^{s}$ is the projection of $X^{\kappa}$ to $X^{s}$, defined by

$$
\pi_{s}^{\kappa}\left(\left\langle x_{\xi}: \xi<\kappa\right\rangle\right)=\left\langle x_{\xi}: \xi \in s\right\rangle
$$

If $\kappa$ is clear from the context, we will write $\pi_{s}$ instead of $\pi_{s}^{\kappa}$. If $s=\{\xi\}$, then we write $\pi_{\xi}$ instead of $\pi_{\{\xi\}}$. A map $f: X^{\kappa} \rightarrow Z$ depends on at most one $(\alpha-t h)$ coordinate if there is $g: X \rightarrow Z$ such that $f\left(\left\langle x_{\xi}\right\rangle_{\xi<\kappa}\right)=g\left(x_{\alpha}\right)$, i.e., $f=g \circ \pi_{\alpha}$.

In all results below, $\kappa$ is an arbitrary cardinal, and all maps are assumed to be continuous.

Theorem 5. Assume $f:\left(\mathbb{N}^{*}\right)^{\kappa} \rightarrow \mathbb{N}^{*}$. Then $\left(\mathbb{N}^{*}\right)^{\kappa}$ can be covered by finitely many clopen sets such that $f$ depends on at most one coordinate on each of them. In particular, there is a finite $s \subseteq \kappa$ and a continuous map $f_{1}:\left(\mathbb{N}^{*}\right)^{s} \rightarrow \mathbb{N}^{*}$ such that the diagram

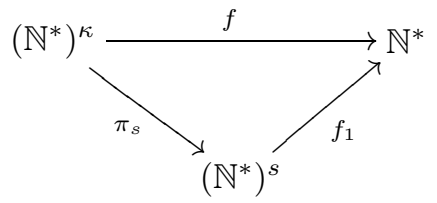

commutes.

Proof. This is a special case of [1 Theorem 3] when $X=Z=\mathbb{N}^{*}$.

Corollary 6. The following are equivalent for every cardinal $\lambda$ :

(1) $\left(\mathbb{N}^{*}\right)^{\kappa} \rightarrow\left(\mathbb{N}^{*}\right)^{\lambda}$ for some $\kappa<\lambda$.

(2) $\left(\mathbb{N}^{*}\right)^{n} \rightarrow\left(\mathbb{N}^{*}\right)^{\lambda}$ for some finite $n<\lambda$.

Proof. We will prove only the nontrivial direction; note that we may assume $\lambda$ is infinite. Assume $f:\left(\mathbb{N}^{*}\right)^{\kappa} \rightarrow\left(\mathbb{N}^{*}\right)^{\lambda}$ is a surjection. By Theorem 5 , for each $\xi<\lambda$ there is a finite $s_{\xi} \subseteq \kappa$ such that $\pi_{\xi} \circ f$ depends only on coordinates in $s_{\xi}$. By a counting argument, there is $A \subseteq \lambda$ of size $\lambda$ and a finite $s \subseteq \kappa$ such that $s_{\xi}=s$ for all $\xi \in A$. Hence there is a map $g:\left(\mathbb{N}^{*}\right)^{s} \rightarrow\left(\mathbb{N}^{*}\right)^{A}$ such that $g \circ \pi_{s}^{\kappa}=\pi_{A} \circ f$. Since the map on the right-hand side is surjective, so is $g$. This concludes the proof.

Proof of Theorem Q Implications $(4) \Rightarrow(1) \Rightarrow(2) \Rightarrow(3)$ are trivial. The equivalence of (4) and (1) follows from Theorem 3 proved below. Thus it only remains to prove (3) implies (1).

Assume $\kappa<\lambda$ and $f:\left(\mathbb{N}^{*}\right)^{\kappa} \rightarrow\left(\mathbb{N}^{*}\right)^{\lambda}$ is a surjection. By Corollary 6 , we may assume $\kappa$ is finite, say $\kappa=n$. We may also assume $\lambda=n+1$. By applying Theorem 5 to $\pi_{i} \circ f$ for all $i<n+1$ and refining the obtained clopen coverings, we can cover $\left(\mathbb{N}^{*}\right)^{n}$ by finitely many clopen sets so that $\pi_{i} \circ f$ depends on at most one coordinate on each of them for each $i<n+1$. The image of at least one of these clopen sets, call it $U$, includes a nonempty clopen subset, call it $V$, of $\left(\mathbb{N}^{*}\right)^{n+1}$. There are $i<j<n+1$ and $k<n$ such that both $\pi_{i} \circ f$ and $\pi_{j} \circ f$ depend at most on the $k$ th coordinate on $U$. Since $V$ is clopen, its image under the projection 
$\vec{y} \mapsto\left\langle\pi_{i}(\vec{y}), \pi_{j}(\vec{y})\right\rangle$ is a clopen subset, call it $V^{\prime}$, of $\left(\mathbb{N}^{*}\right)^{2}$. Let $h_{l}: U \rightarrow \mathbb{N}^{*}$ be such that $\pi_{l} \circ f=h_{l} \circ \pi_{k}$ for $l \in\{i, j\}$ on $U$. Pick $\langle\mathcal{U}, \mathcal{V}\rangle \in V^{\prime}$, and define $h: \mathbb{N}^{*} \rightarrow V$ by

$$
h(\vec{x})= \begin{cases}\left\langle h_{i}(\vec{x}), h_{j}(\vec{x})\right\rangle, & \text { if }\left\langle h_{i}(\vec{x}), h_{j}(\vec{x})\right\rangle \in V^{\prime}, \\ \langle\mathcal{U}, \mathcal{V}\rangle, & \text { otherwise. }\end{cases}
$$

This is a continuous surjection from $\mathbb{N}^{*}$ onto $V^{\prime}$; but $V^{\prime}$ is homeomorphic to $\left(\mathbb{N}^{*}\right)^{2}$, and this concludes the proof.

Proof of Theorem [3. We prove only the nontrivial direction. Let $f_{i}: X \rightarrow X(i \in$ $\{1,2\})$ be continuous maps such that $x \mapsto\left(f_{1}(x), f_{2}(x)\right)$ maps $X$ onto $X^{2}$. Define $g_{n}: X \rightarrow X(n \in \mathbb{N})$ as follows:

$$
\begin{aligned}
& g_{1}=f_{1}, \\
& g_{2}=f_{1} \circ f_{2}, \\
& g_{3}=f_{1} \circ f_{2} \circ f_{2}, \\
& g_{n}=f_{1} \circ \underbrace{f_{2} \circ \cdots \circ f_{2}}_{n-1 \text { times }}, \quad \text { for } n \in \mathbb{N} .
\end{aligned}
$$

We claim that $x \mapsto\left(g_{n}(x)\right)_{n=1}^{\infty}$ maps $X$ onto $X^{\mathbb{N}}$. Since $X$ is compact, it suffices to show that the image of $X$ under this map is dense in $X^{\mathbb{N}}$, and it turn it suffices to show that for every $n \in \mathbb{N}$ and $\vec{y}=\left(y_{i}\right)_{i=1}^{n}$ in $X^{n}$ there is an $x \in X$ such that $\vec{z}=f(x)$ satisfies $z_{i}=y_{i}$ for all $i \leq n$. Equivalently, we need to prove that for every $n$-tuple $\left(y_{1}, \ldots, y_{n}\right)$ there is an $x \in X$ such that $g_{i}(x)=y_{i}$ for $i \leq n$. We prove this by induction on $n$.

For $n=2$, find $x^{\prime} \in X$ such that $f_{1}\left(x^{\prime}\right)=y_{2}$ (possible, since $f_{1}$ is onto), and then, by using the fact that $x \mapsto\left(f_{1}(x), f_{2}(x)\right)$ maps $X$ onto $X^{2}$, find $x \in X$ such that $f_{1}(x)=y_{1}$ and $f_{1}(x)=x^{\prime}$. Then we have $g_{2}(x)=f_{1}\left(f_{2}(x)\right)=f_{1}\left(x^{\prime}\right)=y_{2}$.

Assume the claim is true for $n$ and prove it for $n+1$. By the induction hypothesis, find $x^{\prime} \in X$ such that $g_{i}\left(x^{\prime}\right)=y_{i+1}$, for $1 \leq i \leq n$. Now pick $x$ such that $f_{1}(x)=y_{1}$ and $f_{2}(x)=x^{\prime}$. Then for $2 \leq i \leq n+1$ we have $g_{i}(x)=g_{i-1}\left(f_{2}(x)\right)=g_{i-1}\left(x^{\prime}\right)=y_{i}$, and this completes the proof.

By simple cardinal arithmetic, Theorem 3 implies that a compactum of size strictly less than the continuum does not map onto its own square. The compactness assumption cannot be omitted from Theorem 3 since $X=\mathbb{N}$ maps onto $\mathbb{N}^{2}$ but not onto $\mathbb{N}^{\mathbb{N}}$. J. van Mill points out that the version of this result in which $X^{2}$ and $X^{\mathbb{N}}$ are required to be homeomorphic to $X$ is false by [3].

S. Solecki [7] has pointed out that the above construction of the maps $g_{n}$ appears in 6. In this paper Sierpiński proves that if $x \mapsto\left(f_{1}(x), f_{2}(x)\right)$ maps [0,1] onto $[0,1]^{2}$, then $x \mapsto\left\langle g_{n}(x): n \in \mathbb{N}\right\rangle$ maps $[0,1]$ onto $[0,1]^{\mathbb{N}}$. Although [6] does not use any assumptions beyond the compactness of $[0,1]$, Theorem 3 was not stated in this paper.

Definition 7. Let $\mu$ be the smallest cardinal such that $\mathbb{N}^{*} \operatorname{does}$ not map onto $\left(\mathbb{N}^{*}\right)^{\mu}$.

Note that $\mu$ is at least two, and since the weight of $\mathbb{N}^{*}$ is equal to $\mathfrak{c}, \mu$ is at most $\mathfrak{c}^{+}$. (Recall that weight of a topological space is the minimal size of its base, and that the weight of a continuous image of a compact space does not exceed the weight of the original space.) As pointed out above, $\mu$ can assume either of these two values: by [5], Continuum Hypothesis implies that $\mu=\mathfrak{c}^{+}$while in [2] Just has obtained a forcing extension in which $\mu=2$. By Theorem $2, \mu>2$ implies $\mu \geq \aleph_{1}$. 
Proof of Theorem 4. Let $\mu$ be as in Definition 7. Then $\kappa<\lambda<\mu$ implies $\left(\mathbb{N}^{*}\right)^{\kappa} \rightarrow$ $\mathbb{N}^{*} \rightarrow\left(\mathbb{N}^{*}\right)^{\lambda}$, hence (2) implies (1). To prove (1) implies (2), let us assume $f:\left(\mathbb{N}^{*}\right)^{\kappa} \rightarrow\left(\mathbb{N}^{*}\right)^{\lambda}$ is a surjection and $\kappa<\lambda$. By Corollary [6, we may assume $\kappa$ is finite. By Theorem [2, $\mathbb{N}^{*}$ maps onto $\left(\mathbb{N}^{*}\right)^{\kappa}$, hence by composing we have $\mathbb{N}^{*} \rightarrow\left(\mathbb{N}^{*}\right)^{\lambda}$.

Question 8. Which of the following are relatively consistent with the usual axioms of Set Theory:

(a) $\mu$ is neither 2 nor $\mathbf{c}^{+}$,

(b) $\mu=\aleph_{1}$,

(c) $\mu$ is a limit cardinal.

The following variation on Theorem 3 shows that $\mu$ has to be regular.

Theorem 9. Let $X$ be an arbitrary topological space, and assume $\kappa$ is a singular cardinal such that $X$ maps onto $X^{\lambda}$ for all $\lambda<\kappa$. Then $X$ maps onto $X^{\kappa}$.

Proof. Let $\kappa_{\alpha}(\alpha<\operatorname{cf}(\kappa))$ be a strictly increasing sequence of cardinals with supremum equal to $\kappa$. For $\eta<\kappa$ let $f_{\eta}: X \rightarrow X$ be continuous maps such that $x \mapsto$ $\left(f_{\eta}(x)\right)_{\eta \in\left[\kappa_{\alpha}, \kappa_{\alpha+1}\right)}$ maps $X$ onto $X^{\left[\kappa_{\alpha}, \kappa_{\alpha+1}\right)}$. Define $g_{\eta}: X \rightarrow X(\operatorname{cf}(\kappa) \leq \eta<\kappa)$ by

$$
g_{\eta}=f_{\eta} \circ f_{\alpha} \text { if } \eta \in\left[\kappa_{\alpha}, \kappa_{\alpha+1}\right) .
$$

We claim that $x \mapsto\left(g_{\eta}(x)\right)_{\eta=\kappa_{0}}^{\kappa}$ maps $X$ onto $X^{\left[\kappa_{0}, \kappa\right)}$. Since the latter power is homeomorphic to $X^{\kappa}$, this will conclude the proof. Fix $\vec{y}=\left(y_{\eta}\right)_{\eta=\kappa_{0}}^{\kappa}$. Let $x_{\alpha}(\alpha<\operatorname{cf}(\kappa))$ be such that $f_{\eta}\left(x_{\alpha}\right)=y_{\eta}$ for $\eta \in\left[\kappa_{\alpha}, \kappa_{\alpha+1}\right)$. Now let $x \in X$ be such that $f_{\alpha}(x)=x_{\alpha}$ for all $\alpha<\operatorname{cf}(\kappa)$. Then if $\eta \in\left[\kappa_{\alpha}, \kappa_{\alpha+1}\right)$, we have $g_{\eta}(x)=f_{\eta}\left(f_{\alpha}(x)\right)=f_{\eta}\left(x_{\alpha}\right)=y_{\eta}$, as required.

Question 10. Is it true that if $X$ is a compact space and $\kappa$ is the supremum of all $\lambda$ such that $X$ maps onto $X^{\lambda}$, then $X$ maps onto $X^{\kappa}$ ?

\section{REFERENCES}

[1] I. Farah. Dimension phenomena associated with $\beta \mathbb{N}$-spaces. Topology and its Applications, to appear; available at http://www.math.csi.cuny.edu/ farah.

[2] W. Just. The space $\left(\omega^{*}\right)^{n+1}$ is not always a continuous image of $\left(\omega^{*}\right)^{n}$. Fundamenta Mathematicae, 132:59-72, 1989. MR 90h:54013

[3] J. van Mill. A Peano continuum homeomorphic to its own square but not to its countable infinite product. Proceedings of the American Mathematical Society, 80:703-705, 1980. MR 81k:54061

[4] J. van Mill. An introduction to $\beta \omega$. In Handbook of Set-theoretic topology (K. Kunen and J. Vaughan, editors), pages 503-560. North-Holland, 1984. MR 86f:54027

[5] I.I. Parovičenko. A universal bicompact of weight $\aleph$. Soviet Mathematics Doklady, 4:592-592, 1963.

[6] W. Sierpiński. Remarque sur la courbe péanienne. Wiadomosci Matematyczne, 42:1-3, 1937. reproduced in: W. Sierpiński, Oeuvres Choisies, (1976), volume III, p. 369-371. MR 54:2407

[7] S. Solecki. personal communication. September 2000.

Department of Mathematics, College of Staten Island, 2800 Victory Blvd., Staten Island, New York 10314 and Mathematical Institute, Kneza Mihaila 35, 11000 Beograd, YUGOSLAVIA

E-mail address: ifarah@gc.cuny.edu

URL: http://www. math.csi.cuny.edu/ farah 